\title{
Left atrial appendage closure with the
} Watchman device - new option for patients with atrial fibrillation and high-risk of thromboembolic events

\author{
Anna Olasińska-Wiśniewska, Marek Grygier \\ Poznan University of Medical Sciences, Poland
}

\section{Atrial fibrillation and left atrial appendage}

T The atrial fibrillation (AF) is the most common cardiac tachyarrhythmia. It affects approx. $1 \%$ of general population ${ }^{1}$ and this percentage increases with age affecting about 3,8\% of patients over 60 years and $9 \%$ of patients over 80 years ${ }^{1}$. Stroke is the most debilitating and life-threatening complications of AF. The arrhythmia is associated with even a 5 -fold risk of stroke. ${ }^{2,3}$ The frequency of AF in stroke patients admitted to medical departments ranges from $6,5 \%$ in younger patients (50-59 year) ${ }^{2}$ to over $30 \%$ in octogenarians. ${ }^{2,4,5}$ Thus elderly patients are not only prone to AF, but their stroke risk is also higher. Strokes related to AF are associated with worse prognosis, worse neurological outcome and higher rate of medical complications, including pneumonia, pulmonary oedema and heart failure compared with strokes of other than AF etiology. ${ }^{4}$ The probability of remaining disabled or handicapped is increased by almost $50 \% .^{5}$ The in-hospital and long-term mortality rate are also higher in patients with $\mathrm{AF}^{4,5}$

Blackshear and Odell ${ }^{6}$ reviewed twenty three studies that evaluated the presence and location of left atrial thrombus by transoesophageal echocardiography, autopsy or operation. The analysis revealed that left atrial thrombi occur in left atrial appendage in 91\% of nonrheumatic atrial fibrillation and in $57 \%$ of rheumatic mitral valve disease. Non-rheumatic atrial fibrillation is probably responsible for $15-20 \%$ of cerebrovascular accidents of ischaemic origin. ${ }^{7,8}$

The left atrial appendage (LAA) is a remnant of the primary left atrium which forms during third week of embryonic development. ${ }^{9}$ The proper left atrial cavity develops later and is formed from the outgrowth of the pulmonary veins.

The LAA has a tubular, hooked and trabeculated structure ${ }^{9}$ with considerable heterogeneity among individuals in size, shape, wall thickness and morphology. ${ }^{10}$ It is more distensible than the left atrium proper and may augment haemodynamic function as a decompression chamber by modulating left atrial pressure - volu- me relations in states of increased left atrial pressure and volume overload. ${ }^{9,11}$ The LAA also contains stretch receptors that may regulate thirst [11] and other endocrine cells that produce atrial natriuretic peptide ${ }^{7,9,11}$ and help regulating fluid balance. The cardiocytes of the LAA contain the greatest density of atrial natriuretic peptide granules found in the left atrium. ${ }^{9,11}$ Several authors reported fluid retention after bilateral atrial appendectomy concomitant to maze procedure..$^{12-14}$ In those patients in whom the right atrial appendage was preserved the production of atrial natriuretic peptide was maintained resulting in better diuresis in the postoperative period. ${ }^{12-14}$ The LAA may be also the site of triggers that can induce episodes of AF and of re-entrant drivers that may participate in the AF maintenance. In $A F$, remodeling as well as impaired blood flow occur in left atrial appendage. ${ }^{11}$ These pathological conditions may lead to stasis and thrombus formation. ${ }^{9}$ The degree of stasis in LAA is substantially worse than in the right atrial appendage because of differences in the anatomy and blood flow in both appendages.

\section{Strategies of pharmacological stroke prevention in atrial fibrillation}

According to current guidelines ${ }^{3}$ classic OAC and new OAC (NOAC) are recommended to prevent thromboembolic events in atrial fibrillation patients. The $\mathrm{CHA}_{2} \mathrm{D}$ $\mathrm{S}_{2}$ VASc score was implemented for stroke risk assessment and to guide treatment choice. Stroke in the past and age over 75 years, based on the $\mathrm{CHA}_{2} \mathrm{DS}_{2} \mathrm{VASc}$ score, are two factors strong enough to start OAC therapy in patients with AF. The decision to begin therapy must stay in balance with risk of major bleeding, especially intracranial, which is the most serious complication of this therapy with a high risk of disability and death. ${ }^{3}$ Therefore HASBLED score should be also calculated in each patient to evaluate the risk of bleeding.

There are however even more difficulties associated with $\mathrm{OAC}$, including drug interactions, dietary restriction, poor patient adherence to treatment, labile interna- 
tional normalized ratio (INR) and problematic decisions during urgent invasive procedures. ${ }^{8}$ The majority of strokes in patients who started OAC occur in subjects who have discontinued OAC or whose INR is subtherapeutic. ${ }^{15}$ Moreover, several studies ${ }^{5,16}$ proved that OAC therapy is not properly implemented. Euro Heart Survey Investigators ${ }^{16}$ showed that, despite strong recommendations, OAC therapy was properly prescribed only in $60 \%$ of high risk patients, whereas $28 \%$ were undertreated and $11 \%$ overtreated. Similar results were presented in systematic review of 54 studies performed by Ogilvie et al. ${ }^{17}$ with treatment level ranging from $39 \%$ to $92,3 \%$ of high risk patients based on the CHADS2 risk score. High discontinuation rate (30\%) was also underlined $^{17}$. Moreover, there are several contraindication for OAC, including evidence of active bleeding, history or predisposition to intracranial bleeding, uncontrolled severe hypertension, recent brain, eye or spinal cord surgery or injury, propensity for recurrent falling, inability for INR monitoring, and patient non-compliance.

The NOAC have shown non-inferiority compared with classical OAC and better safety limiting the number of intracranial hemorrhage ${ }^{18,19}$. Nevertheless, many problems with oral anticoagulation remained unresolved. Dabigatran and rivaroxaban are contraindicated in severe kidney disease (with creatinine clearance lower than $30 \mathrm{~mL} / \mathrm{min}$ ), and the dose should be reduced in the presence of high bleeding risk (HAS-BLED score $\geq 3$ ), moderate kidney disease (with creatinine clearance 30-49 $\mathrm{mL} / \mathrm{min}$ ), as well as in elderly patients ( $\geq 80$ years) and if concomitant use of interacting drug (werapamil) is necessary for dabigatran. The risk of major bleeding is similar among NOAC and estimated as 3,36-3,6\% per year during rivaroxaban therapy $y^{18,19}, 3,4 \%$ per year during warfarin therapy ${ }^{19}$ and $2,71 \%$ per year during treatment with dabigatran $110 \mathrm{mg}$ daily and $3,11 \%$ per day with the daily dose $150 \mathrm{mg}$ of dabigatran ${ }^{18}$. ROCKET-AF stu$d y^{19}$ revealed that rates of intracranial hemorrhage were significantly lower in the rivaroxaban group than in the warfarin group ( $0,5 \%$ vs. $0,7 \%$ per year). However major bleeding from a gastrointestinal site was more common in the rivaroxaban group $(3,2 \%)$, as compared with warfarin group $(2,2 \%)$.

\section{Left atrial appendage closure as alternative to pharmacological therapy}

Though there are several pharmacological antithrombotic possibilities, some groups of patients with several contraindications, especially high risk of bleeding and with history of bleeding complications, cannot be offered any of them. Therefore, LAA closure may be an attractive alternative. Attempts to decrease risk of LAA thrombus embolisms resulted in development of surgical excision and percutaneous LAA occlusion techniques. James $\mathrm{Cox}^{7}$, on the basis of surgical studies and his own observations, concludes that removal or proper closure of the LAA at surgery reduces the risk of perioperative and long-term stroke. According to current guidelines ${ }^{3}$ LAA surgical excision may be considered in patients undergoing open heart surgery (Ilb C). Inter-
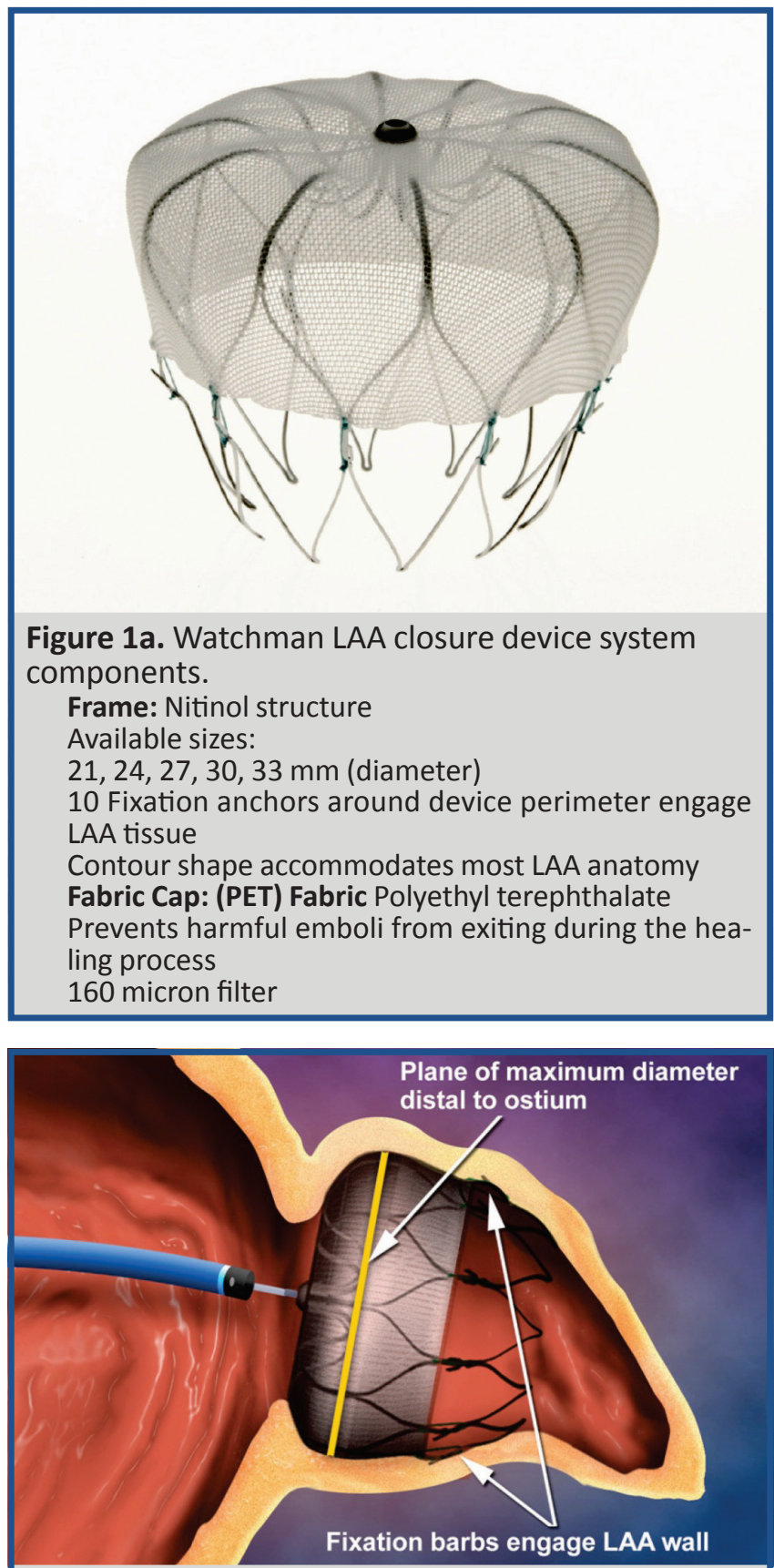

Figure 1b. Optimal position of Watchman device during deployment.

ventional percutaneous LAA closure may be considered in patients with a high stroke risk and contraindications for long-term oral anticoagulation. The LAA closure devices are designed to seal the neck of LAA and reduce thrombus embolization ${ }^{20}$.

Currently, two LAA closure devices are available for clinical use - Watchman ${ }^{\mathrm{TM}}$ left atrial appendage closure device (Watchman device) (Boston Scientific) and Amplatzer ${ }^{\mathrm{TM}}$ Cardiac Plug (Amplatzer device) (St. Jude Medical).

The Watchman device was introduced in 2005. It was designed to be permanently implanted at or slightly distal to the ostium of LAA to trap thrombus before it exits the LAA. The Watchman LAA Closure Technology consists of the Watchman transseptal access system, delivery catheter and an implantable device. The Watchman device is a self-expanding nitinol frame 

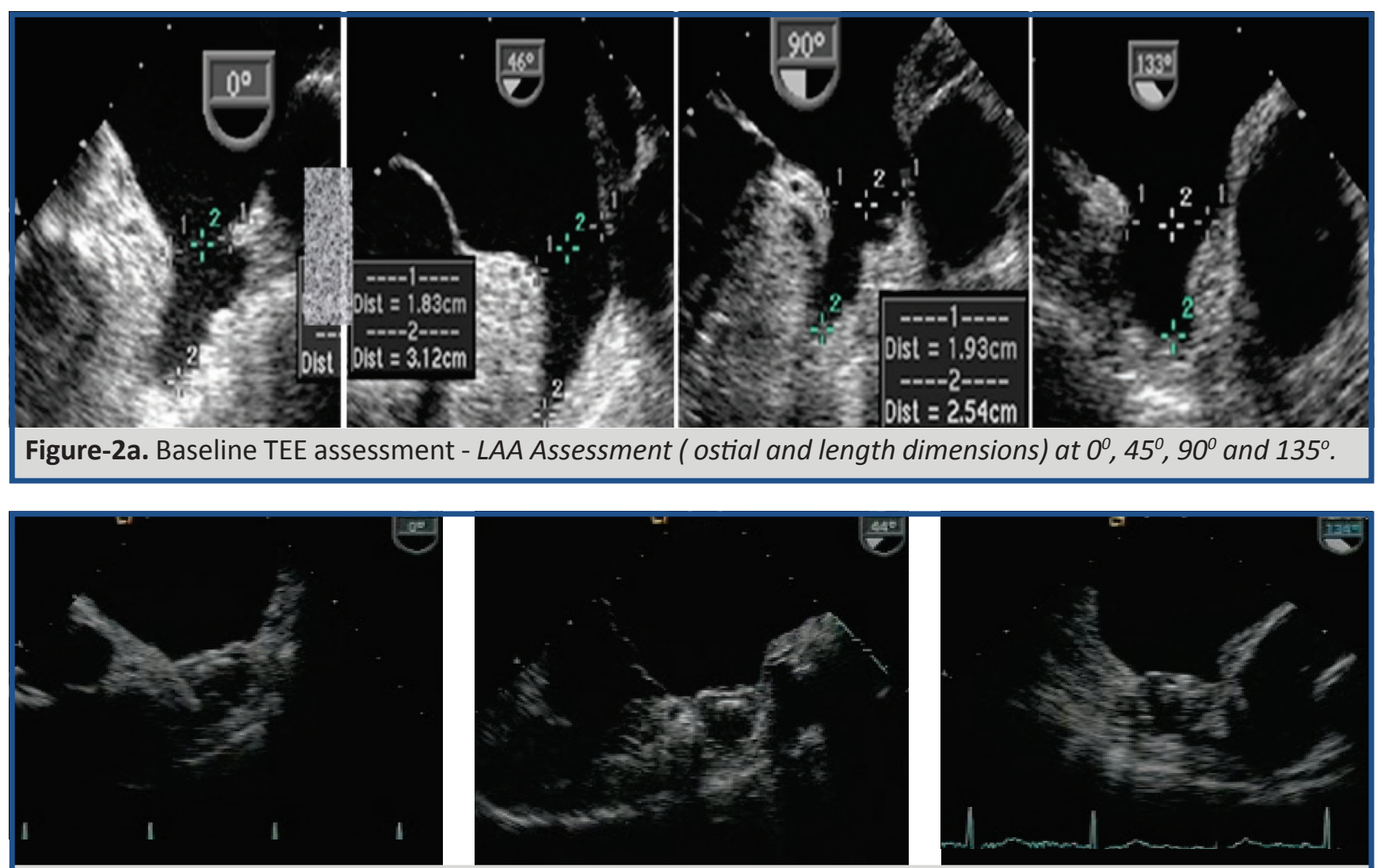

Figure-2b. TEE during procedure - optimal position of Watchman device in the LAA is confirmed by PASS criteria:

1. device is distal to or at the ostium of the LAA;

2. fixation anchors are engaged an device is stable;

3. device is compressed at least $8-20 \%$ of original size;

4. device spans ostium, all lobes of LAA are covered (no residual flow noted around device).

structure with fixation barbs and a permeable polyester fabric cover (Fig-1a and Fig-1b). It is available in 5 sizes $(21-33 \mathrm{~mm})$ and is preloaded within a delivery catheter.

Several studies have shown the feasibility of percutaneous LAA occlusion ${ }^{20-25}$. The first randomized study, the Percutaneous Closure of the Left Atrial Appendage versus Warfarin Therapy for Prevention of Stroke in Patients with Atrial Fibrillation (PROTECT-AF) trail ${ }^{22}$, evaluated the efficacy and safety of the Watchman device compared with standard warfarin therapy. The trial revealed that the efficacy of percutaneous closure of the LAA with the Watchman device is non-inferior to ongoing warfarin therapy with regard to prevention of stroke, systemic embolism, and cardiovascular death. Moreover, the newest analysis of 5 year follow-up of the PROTECT-AF trial revealed significant reduction in cardiovascular (60\%) and all-cause mortality (34\%) in patients treated with the Watchman device compared with warfarin group ${ }^{26}$.

The data from the PROTECT-AF study were confirmed by CAP Registry ${ }^{27}$ and PREVAIL study ${ }^{28}$ which also showed decreased procedure time, improved implant success and procedure/device related safety with increased operator experience. It must be pointed out that these 3 studies were performed in patients who were eligible to take warfarin.

The ASAP study ${ }^{29}$ evaluated the safety and feasibility of the Watchman device for the treatment of non-valvular atrial fibrillation in patients with a contraindication to warfarin. The study showed that Watchman implantation for warfarin contraindicated AF patients is feasible, associated with low, but manageable, rate of device thrombus and decreases the rate of stroke by $77 \%$.

The U.S. Food and Drug Administration (FDA) Circulatory System Devices Panel of the Medical Devices Advisory Committee voted on Dec. 11, 2013 favourably by a majority (13 to 1 ) that the benefits of the WATCHMAN Left Atrial Appendage Closure device outweigh the risks, there is a reasonable assurance that the device is safe and of a reasonable assurance of efficacy. The final decision and approval from the FDA is expected in the first half of 2014 and this innovative technology will be available to patients with AF at higher risk for stroke who need an alternative to long-term warfarin therapy also in USA.

The data regarding the clinical usage of the Amplatzer Cardiac Plug are based on reports of single-centre experience and registries ${ }^{23,25}$. Up to now there are no randomized trials comparing Amplatzer device with oral anticoagulants, thus, in authors opinion, their usage should be for now limited to clinical trials.

\section{Left atrial appendage closure procedure with the Watchman device}

The procedures may be done under general (preferably) or local anesthesia and with the use of transoesophageal echocardiography and fluoroscopy in a 


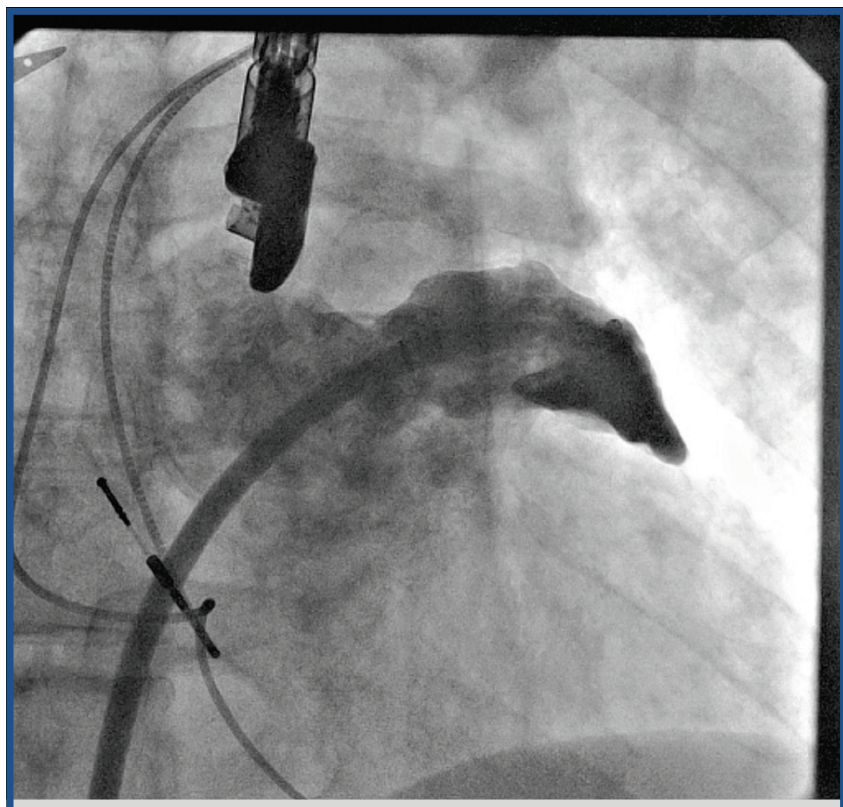

Figure 3a. Fluroscopic visualization of left atrial appendage and right atrium through guiding catheter located in the ostium of left atrial appendage

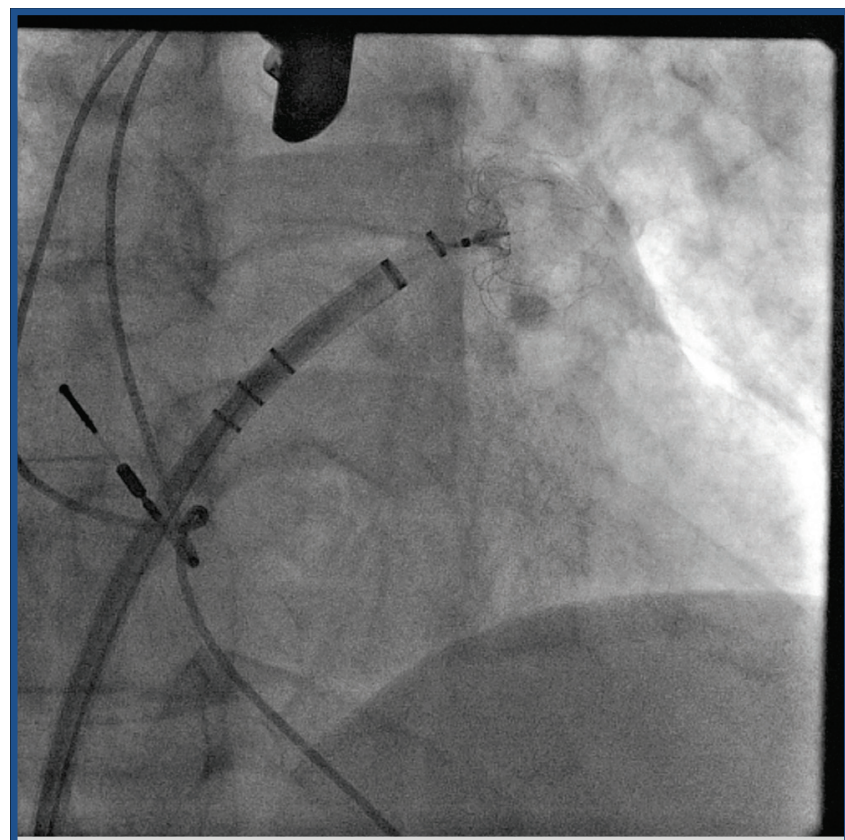

Figure 3c. The Watchman device fully opened in the left atrial appendage

catheterization laboratory (Fig. 2a-2b; Fig 3a-3d). Vascular access is obtained with puncture of femoral vein. After cannulation of the femoral vein, a mid-low and posterior transseptal puncture is performed under transoesophageal echocardiography guidance using conventional transeptal needle and delivery sheat. Heparin is then given to keep an ACT above $250 \mathrm{sec}$. Then, the Watchman Access Sheath and Dilator are advanced over a guidewire into the left atrium. The LAA is engaged with a $5 \mathrm{~F}-6 \mathrm{~F}$ pigtail catheter to perform selective angiograms. The Access Sheath is then carefully advanced into the distal portion of the LAA over a pigtail catheter. The LAA morphology is then carefully analysed in both angiograms and transoesophageal echocardio-

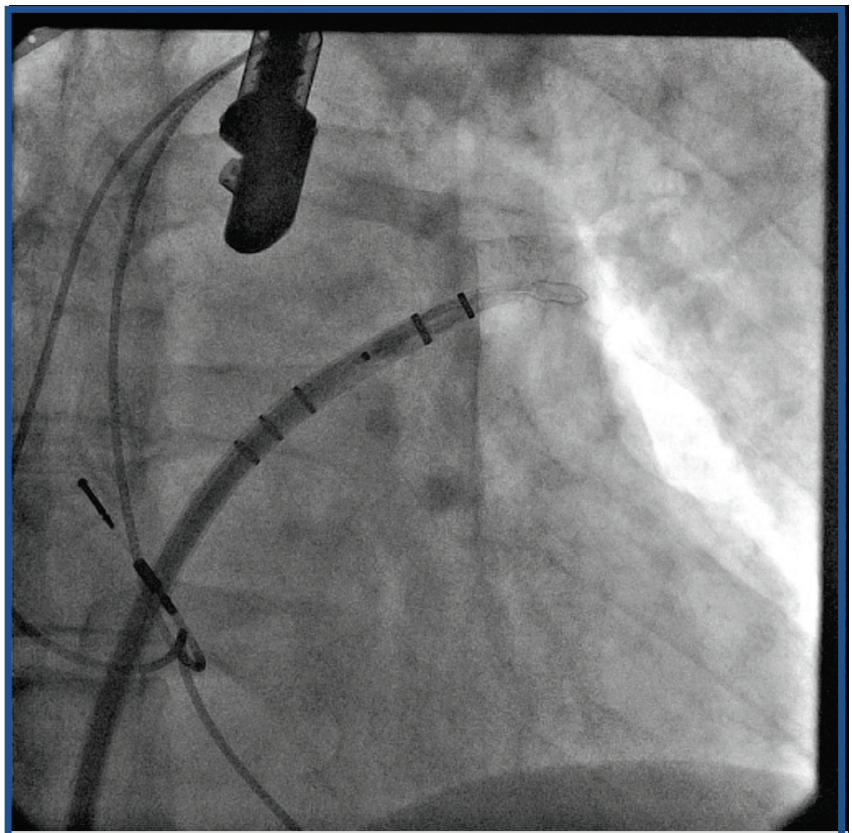

Figure $3 b$. The Watchman device partially opened in the left atrial appendage

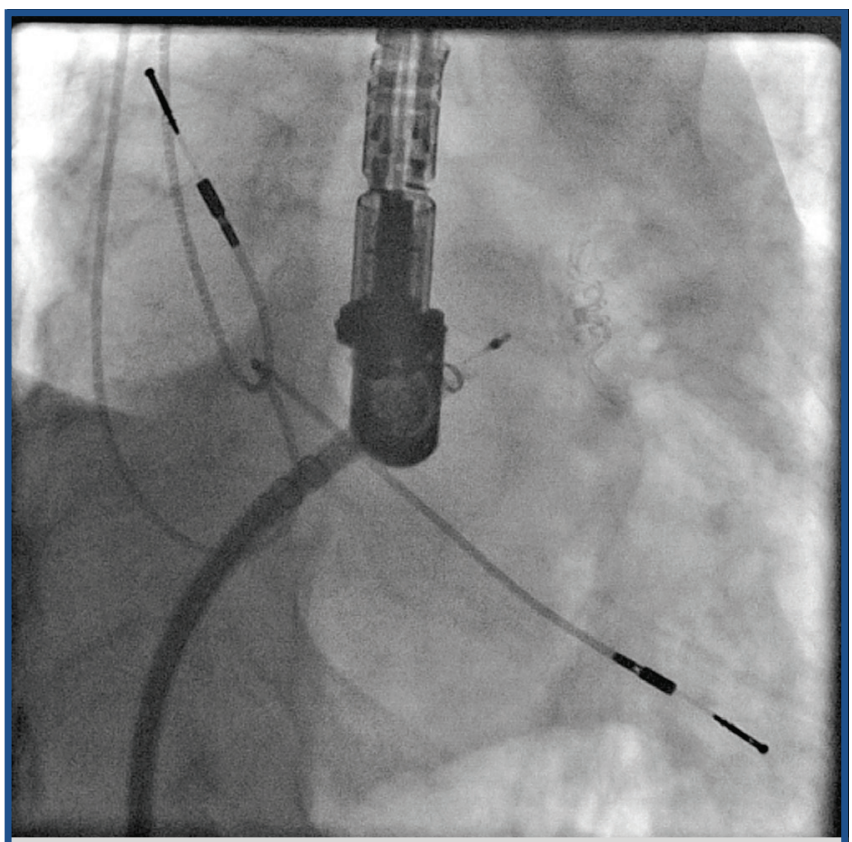

Figure $\mathbf{3 d}$. The Watchman device immediately after release from delivery cable

graphy to determine which size of the Watchman device should be implanted. Precise measurements of LAA are carried out in transoesophageal echocardiography in 0, 45, 90 and 135 degree. The Watchman Delivery System is prepared, inserted into the Access Sheath, and slowly advanced under fluoroscopic guidance. The Watchman Device is then deployed into the LAA. The device release criteria are confirmed via fluoroscopy and TEE prior to releasing the device.

Periprocedural complications are the major problem of interventional LAA closure, especially during learning phase. The overall complication rate could be as high as $8,7-11,7 \% .^{20,22,27,29}$ Most often are pericardial effusion $(1,1-5,0 \%)$, cardiac tamponade $(1,1-1,3 \%)$, major blee- 
ding $(3,5 \%)$, puncture site complications ( $2 \%)$, thrombus formation on the device $(0,7-1,1 \%)$ or on the sheath $(0,6 \%)$, device embolization $(0,6-1,7 \%)$, air embolization $(1,7 \%)$, ischemic stroke $(0,7-1,1 \%)$ or hemorrhagic stroke $(0,2 \%)$ or TIA $(0,6 \%) .^{20,22,27,29}$ In PROTECT-AF trial ${ }^{22}$ twenty one of 463 subjects assigned to the intervention group died during the study, however no deaths were deemed related to the Watchman device. Similarly no deaths device or procedure related were reported in the ASAP study ${ }^{29}$ and in Matsuo el al. study ${ }^{20}$. According to results of CAP and PREVAIL ${ }^{27,28}$ there is a significant improvement in the safety of Watchman left atrial appendage closure with increased operator experience. In the study performed by Reddy et al. ${ }^{27}$ the cohort included 542 patients of the PROTECT-AF trial and a subsequent registry of 460 patients undergoing Watchman implantation (Continued Access Protocol - CAP Registry). A remarkable reduction in the rate of procedure- or device-related safety events was observed, including reduction in procedural time (mean $62 \pm 34 \mathrm{mi}$ nutes in PROTECT-AF and mean 50 \pm 21 minutes in CAP), the rate of serious pericardial effusion ( $5 \%$ in PROTECTAF to $2,2 \%$ in CAP), device embolization (3 cases in PROTECT-AF and none in CAP), and periprocedural stroke rates $(0,9 \%$ in PROTECT-AF and no strokes in CAP). The successfulness of implantation increased from $89,5 \%$ in PROTECT-AF to $95 \%$ in CAP. Pericardial effusion was the major component of early safety events in PROTECT-AF. Based on the review of procedural details, fluoroscopy and TEE imaging, a variety of causes of pericardial effusion were recognized, ranging from being the result of transseptal puncture, the delivery sheath, or the actual manipulation of the Watchman device itself. The rate of serious pericardial effusion in CAP was less than half that seen in PROTECT-AF. There was also experience-related improvement in periprocedural stroke rate. This complication was largely related to the inadvertent introduction of air entrapped within the sheath to the systemic circulation during the procedure. With careful sheath management, there have been no periprocedural strokes in the CAP registry. Similarly, procedural protocol changes implemented over the study period, resulted in decrease in device embolization rate. Importantly, the safety events rates in the Watchman group had a skewed distribution with a large initial event rate, and subsequent rate during follow-up, while the safety events in the warfarin group occurred at approximately constant rates over time and would be expected to continue to accumulate linearly potentially beyond the end of the study period ${ }^{27}$. Not surprisingly, in Reddy et al. ${ }^{24}$ analysis the exclusion of periprocedural adverse events favored the device strategy. After exclusion of events that occurred on the day of device deployment, fewer patients experienced the primary efficacy events in device than in the control warfarin group (postprocedure, $2,5 \%$ per year versus $4,3 \%$ per year). The similar result was found when analysis was confined to patients who stopped warfarin after successful device deployment (2,3\% per year versus $4,1 \%$ per year), as well as those who completed therapy with warfarin and clopidogrel and were only taking aspirin $(2,3 \%$ per year versus $4,1 \%$ per year in control group). These analyses suggest that after successful procedure, the LAA device is more effective than continued warfarin therapy.

Post-interventional anti-thrombotic treatment schedule is not clearly established yet. PROTECT-AF [22] patients were treated with warfarin for 45 days to facilitate device endothelialisation. Warfarin was then stopped depending on the result of the transoesophageal echocardiography 45 days after the procedure (lack of flow around the device) and pharmacotherapy was continued with clopidogrel $75 \mathrm{mg}$ daily for 6 months and aspirin (81-325 mg daily) for long term use. The non-randomised ASAP study29] showed that treatment with aspirin and clopidogrel in patients with contraindications for even short term anticoagulation is feasible, safe and effective.

\section{Conclusions}

The clinical data demonstrate that the WATCHMAN LAA Closure Technology is a safe and effective alternative to warfarin therapy in reducing the risk of stroke, cardiovascular death and systemic thromboembolism in patients with non-valvular atrial fibrillation. It should be especially strongly considered in patients who have contraindications for oral anticoagulation or have complications associated with such treatment.

\section{References:}

1. Go AS, Hylek EM, Phillips KA et al. Prevalence of diagnosed atrial fibrillation in adults: national implications for rhythm management and stroke prevention: the AnTicoagulation and Risk Factors in Atrial Fibrillation (ATRIA) Study. JAMA 2001; 285: 2370-2375.

2. Wolf PA, Abbott RD, Kannel WB. Atrial fibrillation as an independent risk factor for stroke: the Framingham Study. Stroke 1991; 22: 983-988.

3. Camm AJ, Lip GY, De Caterina R, et al. 2012 focused update of the ESC Guidelines for the management of atrial fibrillation: an update of the 2010 ESC Guidelines for the management of atrial fibriIlation. Developed with the special contribution of the European Heart Rhythm Association. Eur Heart J 2012; 33: 2719-2747.

4. Steger C, Pratter A, Martinek-Bregel M, et al. Stroke patients with atrial fibrillation have a worse prognosis than patients without: data from the Austrian Stroke registry. Eur Heart J 2004; 25: $1734-1740$.

5. Lamassa M, Di Carlo A, Pracucci G, et al. Characteristics, outcome, and care of stroke associated with atrial fibrillation in Europe: data from a multicenter multinational hospital-based registry (The European Community Stroke Project). Stroke 2001; 32:392-398.

6. Blackshear JL, Odell JA. Appendage obliteration to reduce stroke in cardiac surgical patients with atrial fibrillation. Ann Thorac Surg 1996; 61:755-759.

7. Cox JL. Mechanical closure of the left atrial appendage: is it time to be more aggressive? J Thorac Cardiovasc Surg 2013; 146: 1018-1027.e2.

8. Crystal E, Connolly SJ. Role of oral anticoagulation in management of atrial fibrillation. Heart 2004; 90: 813-817

9. Al-Saady NM, Obel OA, Camm AJ. Left atrial appendage: structure, function, and role in thromboembolism. Heart 1999; 82:547-554.

10. Chatterjee S, Alexander JC, Pearson PJ, Feldman T. Left atrial appendage occlusion: lessons learned from surgical and transcatheter experiences. Ann Thorac Surg 2011; 92: 2283-2292.

11. Stöllberger C, Schneider B, Finsterer J. Elimination of the left atrial appendage to prevent stroke or embolism? Anatomic, physiologic, and pathophysiologic considerations. Chest 2003; 124: 2356-2362. 
12. Omari BO, Nelson RJ, Robertson JM. Effect of right atrial appendectomy on the release of atrial natriuretic hormone. J Thorac Cardiovasc Surg 1991; 102: 272-279.

13. Kim KB, Lee $\mathrm{CH}, \mathrm{Kim} \mathrm{CH}, \mathrm{Cha}$ YJ. Effect of the Cox maze procedure on the secretion of atrial natriuretic peptide. J Thorac Cardiovasc Surg 1998; 115: 139-146.

14. Yoshihara F, Nishikimi T, Sasako Y, et al. Preservation of the right atrial appendage improves reduced plasma atrial natriuretic peptide levels after the maze procedure. J Thorac Cardiovasc Surg 2000; 119: 790-794.

15. Wyse DG, Waldo AL, DiMarco JP, et al. Atrial Fibrillation Followup Investigation of Rhythm Management (AFFIRM) Investigators. A comparison of rate control and rhythm control in patients with atrial fibrillation. N Engl J Med 2002; 347: 1825-1833.

16. Nieuwlaat R, Olsson SB, Lip GY, et al.; Euro Heart Survey Investigators. Guideline-adherent antithrombotic treatment is associated with improved outcomes compared with undertreatment in high-risk patients with atrial fibrillation. The Euro Heart Survey on Atrial Fibrillation. Am Heart J 2007; 153:1006-1012.

17. Ogilvie IM, Newton N, Welner SA, Cowell W, Lip GY. Underuse of oral anticoagulants in atrial fibrillation: a systematic review. Am J Med 2010; 123:638-645.e4.

18. Connolly SJ, Ezekowitz MD, Yusuf S, et al.; RE-LY Steering Committee and Investigators. Dabigatran versus warfarin in patients with atrial fibrillation. N Engl J Med 2009; 361:1139-1151.

19. Patel MR, Mahaffey KW, Garg J, et al.; ROCKET AF Investigators Rivaroxaban versus warfarin in nonvalvular atrial fibrillation. $N$ Engl J Med 2011; 365:883-891.

20. Matsuo Y1, Sandri M, Mangner N, et al. Interventional closure of the left atrial appendage for stroke prevention. Circ J 2014; 78: 619-624.

21. Bayard YL, Omran H, Neuzil P, et al. PLAATO (Percutaneous Left Atrial Appendage Transcatheter Occlusion) for prevention of cardioembolic stroke in non-anticoagulation eligible atrial fibriIlation patients: results from the European PLAATO study. EuroIntervention 2010; 6: 220-226.
22. Holmes DR, Reddy VY, Turi ZG, et al.; PROTECT AF Investigators Percutaneous closure of the left atrial appendage versus warfarin therapy for prevention of stroke in patients with atrial fibrillation: a randomised non-inferiority trial. Lancet 2009; 374:534-542.

23. Streb W, Szymała M, Kukulski T, et al. Percutaneous closure of the left atrial appendage using the Amplatzer Cardiac Plug in patients with atrial fibrillation: evaluation of safety and feasibility. Kardiol Pol. 2013;71(1):8-16.

24. Reddy VY, Doshi SK, Sievert H, et al.; PROTECT AF Investigators. Percutaneous left atrial appendage closure for stroke prophylaxis in patients with atrial fibrillation: 2.3-Year Follow-up of the PROTECT AF (Watchman Left Atrial Appendage System for Embolic Protection in Patients with Atrial Fibrillation) Trial. Circulation 2013; 127:720-729.

25. Lam YY, Yip GW, Yu CM, et al. Left atrial appendage closure with AMPLATZER cardiac plug for stroke prevention in atrial fibrillation: initial Asia-Pacific experience. Catheter Cardiovasc Interv 2012; 79:794-800.

26. Long Term Results of PROTECT AF: The Mortality Effects of Left Atrial Appendage Closure Versus Warfarin for Stroke Prophylaxis in AF - Dr Vivek Y Reddy (Mount Sinai School of Medicine, New York, NY) when presenting the data at the Heart Rhythm Society 2013 Scientific Sessions.

27. Reddy VY, Holmes D, Doshi SK, Neuzil P, Kar S. Safety of percutaneous left atrial appendage closure: results from the Watchman Left Atrial Appendage System for Embolic Protection in Patients with AF (PROTECT AF) clinical trial and the Continued Access Registry. Circulation 2011; 123:417-424.

28. http://www.bostonscientific.com/watchman-eu/clinical-data/ prevail-clinical-study.html

29. Reddy VY, Möbius-Winkler S, Miller MA, et al. Left atrial appendage closure with the Watchman device in patients with a contraindication for oral anticoagulation: the ASAP study (ASA Plavix Feasibility Study With Watchman Left Atrial Appendage Closure Technology). J Am Coll Cardiol 2013; 61: 2551-2556. 